\title{
Computer-aided method for calculating animal configurations during social interactions from two-dimensional coordinates of color-marked body parts
}

\author{
P. ŠUSTR \\ Research Institute of Animal Production, Prague, Czech Republic \\ and University of South Bohemia, České Budějovice, Czech Republic \\ M. ŠPINKA \\ Research Institute of Animal Production, Prague, Czech Republic \\ and \\ S. CLOUTIER and R. C. NEWBERRY \\ Washington State University, Pullman, Washington
}

\begin{abstract}
In an experiment investigating the impact of preweaning social experience on later social behavior in pigs, we were interested in the mutual spatial positions of pigs during paired social interactions. To obtain these data, we applied a different colored mark to the head and back of each of 2 pigs per group and videotaped the pigs' interactions. We used the EthoVision tracking system to provide $x, y$ coordinates of the four colored marks every $0.2 \mathrm{sec}$. This paper describes the structure and functioning of a FoxPro program designed to clean the raw data and use it to identify the mutual body positions of the 2 animals at 0.2 -sec intervals. Cleaning the data was achieved by identifying invalid data points and replacing them by interpolations. An algorithm was then applied to extract three variables from the coordinates: (1) whether the two pigs were in body contact; (2) the mutual orientation (parallel, antiparallel, or perpendicular) of the two pigs; and (3) whether the pig in the "active" position made snout contact in front of, or behind, the ear base of the other pig. Using these variables, we were able to identify five interaction types: Pig A attacks, Pig B attacks, undecided head-to-head position, "clinch" resting position, or no contact. To assess the reliability of the automatic system, a randomly chosen 5-min videotaped interaction was scored for mutual positions both visually (by 2 independent observers) and automatically. Good agreement was found between the data from the 2 observers and between each observer's data and the data from the automated system, as assessed using Cohen's kappa coefficients.
\end{abstract}

In animal behavior studies, it can be necessary to obtain a finely time structured record of the movements of animals while they interact. For instance, to assess whether social interactions in canid or rodent species are exploratory, playful, or agonistic, it is useful to track the mutual spatial positions of dyads of animals (Golani, 1992; Golani, Kafkafi, \& Drai, 1999; Moran, Fentress, \& Golani, 1981). During these interactions, the mutual configurations of the 2 animals usually change very quickly, making scoring through direct visual observation impossible. Analyzing animal positions from videotapes or digitized video files is feasible, but extremely time-consuming. If the scoring must be done at a rate of several records per

This work was supported by Grant QD0100 of the Czech Ministry of Agriculture to M.S. , and by Adler and Farrell Funds to R.C.N. Correspondence should be addressed to P. Sustr, Ethology Group, Research Institute of Animal Production, Přátelství 815, 10401 Prague, Uhříněves, Czech Republic (e-mail: sustr@vuzv.cz). second, researchers are effectively prevented from investigating questions that can be addressed only through analysis of large data sets.

Obviously, automated collection of data on the mutual spatial positions of 2 animals would solve the problem. EthoVision (Spink, Tegelenbosch, Buma, \& Noldus, 2001) and other similar applications provide a partial solution since they can track the position and movement of colored spots on animals in video recordings and save the data in the form of $x, y$ coordinates of the spots at predetermined intervals (e.g., every $0.1 \mathrm{sec})$. A researcher can place two different colored marks on the body of each of 2 focal animals, videotape them while engaged in social interaction, and then track the four colored spots using EthoVision. This method produces data that include potential information about the mutual positions and orientations of the 2 participants. To our knowledge, however, no software is currently available to turn the raw data (i.e., the series of $x, y$ coordinates of the four colored marks) 
into variables such as the point of contact, and the angle between the body axes, of the 2 animals. While conducting an experiment on social interactions in young pigs, we decided to develop a system that would accomplish this. This paper describes our solution.

Young pigs engage in a variety of social interactions ranging from obviously playful gambols to fierce and severely injurious fights. It is important to understand factors that determine the type, duration, and intensity of interactions between young pigs. Severe fighting compromises both the welfare and productivity of pigs kept on commercial farms (Fraser, Kramer, Pajor, \& Weary, 1995; Giersing \& Anderson, 1998). On the other hand, social play experience may help pigs to cope better when subsequently encountering situations in which severe fighting might otherwise occur. Besides having practical importance, understanding pig play is interesting from a comparative perspective since pigs are unique among ungulates in having large litters.

In fights between pairs of strangers, the mutual spatial positions of the contestants are important (Rushen \& Pajor, 1987). The attacking pig attempts to achieve a spatial configuration in which it is able to bite the ears, neck, and shoulders of the opponent. The defending pig attempts to protect itself from being bitten by turning away from the attacker. It can also react by turning quickly toward the attacker, trying to achieve an attacking position itself. When the outcome of the fight is not yet decided but the pigs are exhausted, they often rest in a symmetrical, antiparallel position, similar to a "clinch" in boxing. The position taken when 2 interacting pigs make head-to-head contact appears to be more frequent in play fights than during serious fighting.

We designed an experiment to assess the impact of preweaning social experience on the subsequent social behavior of pigs. We manipulated early social play experience in three treatments (standard, reduced, or enriched; eight litters per treatment) and investigated the effect of these preweaning treatments on agonistic and play behavior when the pigs were introduced to strangers in standardized tests after weaning (Newberry, Spinka, \& Cloutier, 2000).

\section{METHOD}

In each test, 2 pigs from one litter were placed for $30 \mathrm{~min}$ in a $2 \times$ $2 \mathrm{~m}$ arena with 2 unfamiliar pigs from a different litter. Each set of 4 pigs was tested on 3 days, starting when the pigs were 32 days old. Approximate length of piglets was $70 \mathrm{~cm}$.

For a proper evaluation of our tests, we needed a fine-grained sequencing of the mutual positions of the pigs. Direct scoring by a human observer was impossible since the mutual orientation of the opponents changed too quickly. From slow-motion video, we estimated that scoring the positions at 0.2 -sec intervals would be sufficient to capture all configurational changes in a given dyad. Scoring from videotapes by pausing every $0.2 \mathrm{sec}$ was possible in principle (since the interactions are primarily two-dimensional, and an overhead camera gives a very good view). However, analyzing just the first $10 \mathrm{~min}$ of an interaction required collection of 3,000 data points, and we wanted to analyze data from 24 sets of 4 pigs, each tested on 3 days. Thus, pausing the tape (or a digitized video file) every $0.2 \mathrm{sec}$ and scoring the mutual positions manually was out of question, too.

We decided to use EthoVision software (Noldus Information Technology, 1997), which is capable of tracking several differently colored objects simultaneously, to track pig interactions recorded on video. Before each test, we marked 1 focal pig from each group of 4 test pigs with a large blue spot on the top of the head and neck, and a large green spot on the back behind the shoulders, using waxbased livestock markers. A 2nd focal pig (from the other litter) was marked with orange on the head and neck, and pink on the back. The other 2 pigs were not marked. Each colored mark was about $15 \times 15 \mathrm{~cm}$ in size, or ca. $15 \times 15$ pixels on videotape. We tried various combinations of colors in pilot trials but found many of them unsuitable because EthoVision was unable to distinguish them from the background (black-painted plywood walls and straw bedding) or confused one color with another. The combination of colors described above proved satisfactory. However, even with this combination, in about 5\%-15\% of the scans (made five times per second), the system either did not find the "object" (i.e., the colored mark) in question or recorded a false location (e.g., a piece of straw was confused with the green mark, a light reflection on the wall was confused with the blue mark, or the colored mark was hidden by another pig). Although EthoVision offers the option of correcting individual tracking points manually, the amount of data to be collected precluded using this option.

\section{Correcting Mistakes in Track Data}

To automate the correction process, we wrote a program in FoxPro, Version 6.0 (Microsoft Corp., 1998) that identif ied and replaced erroneous data. The program achieves this task in three steps (Figures 1 and 2): (1) identifying incorrect data; (2) locating the first correct position of the object after the incorrect data; and (3) replacing the incorrect data with interpolated values.

Identification of incorrect data using a "too-fast" rule. We based our identification of errors on the fact that the speed of movement of a pig had an upper limit and, hence, the colored marks could not move more than a certain distance between 2 data points. However, the speed of pig movement was very variable: When the pig was sprinting, the colored marks could move at a speed that would normally indicate an error in the data. Hence, we imposed two different cutoff points for the speed of a colored mark. If an object moved more than 50 pixels between scans, the 2 nd data point was always labeled as "error." If the object moved between 20 and 50 pixels, the second value was considered correct if the movement was in the same direction as in both the previous and the following 0.2 -sec intervals ("sprint" movement). Otherwise, it was also labeled "error."

Locating the correct position. The "too-fast" rule identifies the cases when the object was recorded in an incorrect location by EthoVision. However, this rule also results in labeling as "error" the 1st data point after the color mark had returned from an incorrect location to the actual current location of the pig because this movement was also "too fast." To identify when the tracking system recovered the correct location of the pig, our program makes a second run through the data set. When it encounters an "error" label, it stores the previous position of the object as the "last correct" position. Then it scans the subsequent data points, looking for the first point (the "first correct" position) fulfilling one of the two following criteria: Either it was located within 20 pixels of the last correct position, or there were 4 data points in a row that were all within 20 pixels of each other. The second possibility was included in the program because the location of the colored mark could flicker around false locations for several data points and the pig could have moved in the meantime to a location relatively distant from the "last correct" position.

Replacement of error data by interpolated values. In a third run through the data, the program replaces all data points located 


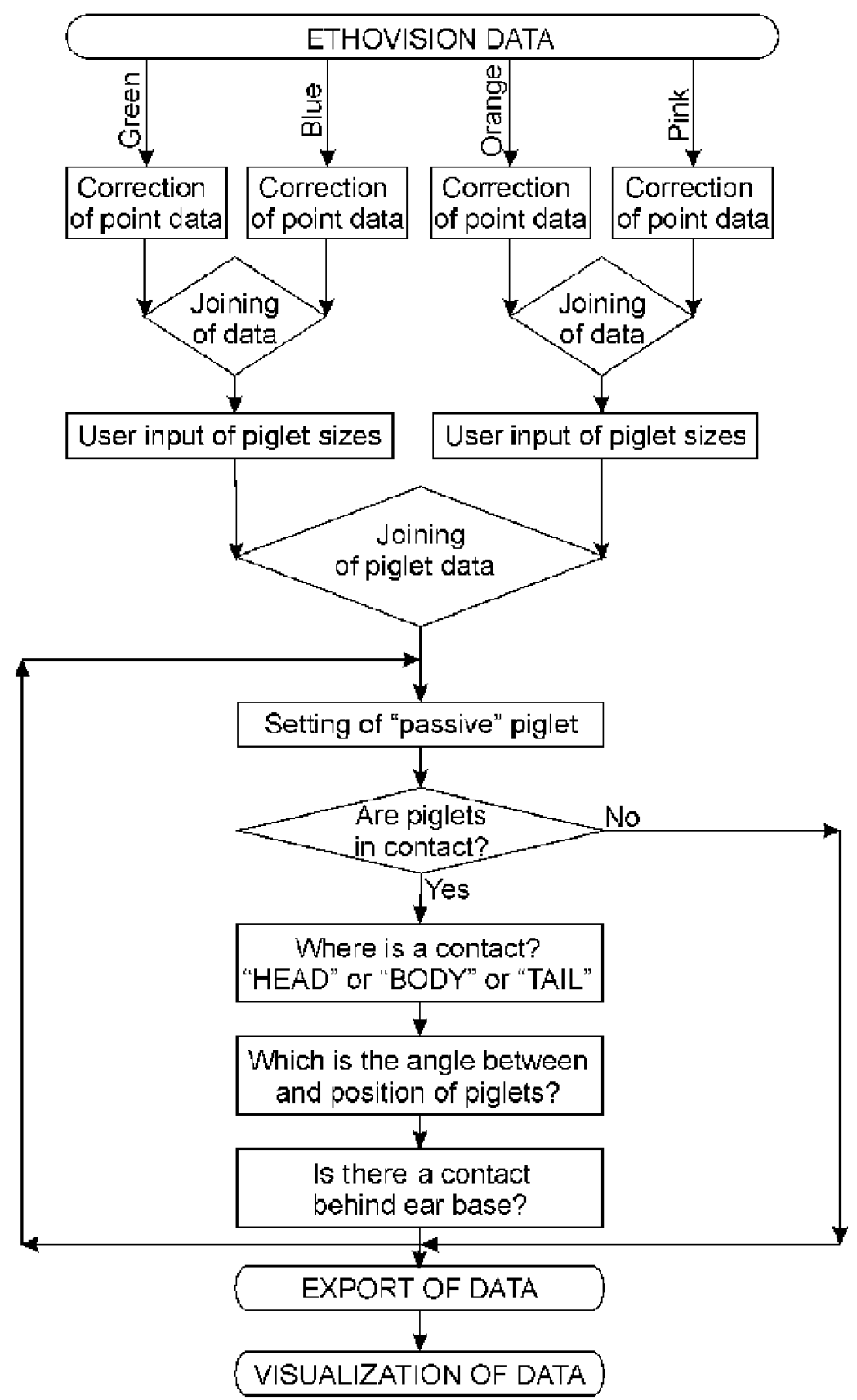

Figure 1. Flow diagram of the described procedure. Process of data correction subroutine is shown in Figure 2.

between the "first correct" and "last correct" positions with linearly interpolated values.

\section{Calculating of Mutual Positions}

To calculate the mutual positions of 2 pigs from the corrected coordinates of the four colored marks, we made the following simplifying assumptions: (1) The bodies of the pigs were assumed not to bend. This assumption was a consequence of the fact that we had just two markers on each piglet, and hence could assess the orientation of the body, but not its flexion. On this assumption, the axes of the 2 pig bodies could be displayed as vectors. (2) The ground projection of the pig was assumed to be a rectangle with an ap- pended semicircle on each end of the rectangle (the "head" and the "tail").

Using these assumptions, our program calculates the locations of the snout, ears, and tail of each marked pig, as well as its ground projection, by extrapolating the "head mark-back mark" vectors in the appropriate directions. To accomplish this task, we determined the following relative lengths for each marked pig: "snout to head mark," "head mark to ears," "head mark to back mark," and "back mark to tail," by measuring them on the video screen. The center of each colored mark was estimated visually for these measurements. We also measured the relative width of each pig's body. These values were used as parameters in the algorithm. 


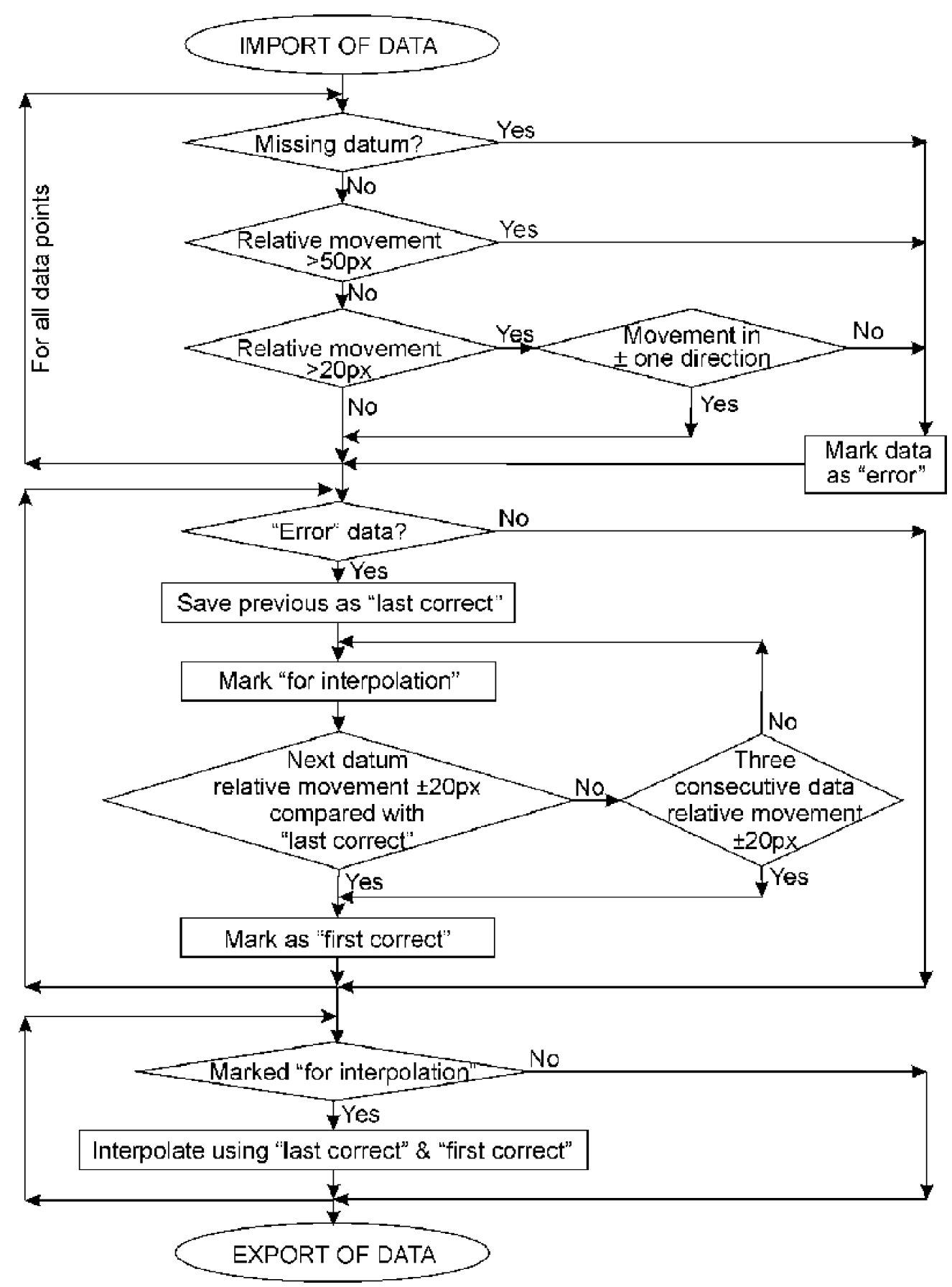

Figure 2. Flow diagram of raw data correction subroutine.

Next, the program determines which of the pigs is "active" at each scan (i.e., having its snout nearer to the body of the 2 nd pig than vice versa) and stores this information as "active pig." The 2-pig configuration is then rotated so that the passive pig has its snout at the $x=0, y=0$ point and its body falls on the negative half of the $y$-axis.

Finally, three variables that describe the mutual positions of the interacting animals are calculated. The first variable, contact, has the value of 1 if the snout coordinates of the active pig are within the ground projection of the body of the other pig, and a value of 0 if otherwise. The second variable, point of contact, distinguishes whether the snout of the active pig makes contact with the passive pig in front of, or behind, the base of the ears. The third variable, the mutual angle of the two bodies, has three possible values: parallel - the two body axes point in the same direction with a maximum divergence of $\pm 45^{\circ}$; antiparallel - the two axes point in opposite directions with a maximum divergence of $\pm 45^{\circ}$; and from side - the active pig's body is pointing perpendicularly toward the body of the passive pig, $\pm 45^{\circ}$. The variables point of contact and angle are calculated only if contact $=1$. With these variables, it is 
Table 1

Classification of Pig Interactions into Five Types According to Variables Extracted From the Tracking Data

\begin{tabular}{|c|c|c|c|c|}
\hline Type of Interaction & Contact & Point of Snout Contact & Angle & Active Pig \\
\hline No interaction & No & Not applicable & Any & Either \\
\hline Head-to-head & Yes & In front of ears & Any & Either \\
\hline Clinch & Yes & Behind ears & Antiparallel & Either \\
\hline BlueGreen attacks & Yes & Behind ears & Parallel or from side & BlueGreen \\
\hline OrangePink attacks & Yes & Behind ears & Parallel or from side & OrangePink \\
\hline
\end{tabular}

possible to classify all mutual positions into interaction types of significance for the winning/losing dynamics of social interactions (Table 1).

\section{Comparison of Visually and Automatically Scored Data}

Reliability in recognizing mutual positions. To assess the reliability of our automated method, a randomly selected 5-min sequence of interactions between 2 color-marked pigs from our experiment was scored for mutual positions both visually and automatically. The 2 pigs were 32 days old, reared under typical swine production conditions, belonged to different litters, and met for the first time during the recording. Visual scoring was performed independently by 2 observers. The videotape was paused every whole second, and the observer recorded in a computer spreadsheet whether the 2 pigs were in contact. If so, then the variables active pig, point of contact, and angle were scored as defined above.

From the 5-min observation, each observer produced a table of 300 records. A comparison was made between the records of the 2 observers to assess interobserver concordance. The records of the observers were also compared with 300 corresponding records produced by the automated system (sampling at 1-sec intervals), which included both noninterpolated and interpolated data. Each record in each of the three data sets was classified into one of five interaction types, based on a combination of the variables contact, point of contact, angle, and active pig, according to the rules specified in Table 1.
The FREQ procedure of the SAS Institute (2000) statistical package was used to calculate the degree of agreement between the $2 \mathrm{ob}-$ servers and the automated system for the variables contact, angle, and type of interaction. The default options of Cicchetti-Allison weight types and table scores were used. For the variable angle, we analyzed only those records in which all three data sets agreed that contact $=1$. The weighted Cohen's kappa coefficients and their confidence limits are presented in Figure 3. For all three variables, the concordance between the automated data and the observers' data was somewhat less than the interobserver concordance. However, the confidence limits of the kappa coefficients overlapped, indicating that the automated system performed at an acceptable level.

Accuracy of interpolated data. To determine whether the replacement of missing or erroneous values by interpolated data was successful, we compared the 300-record data sets produced by the automated system and those produced by Observer 1 . We calculated kappa coefficients for both the noninterpolated data points (i.e., those records in which the system captured the position of all four color markers correctly, $n=132$ ) and the interpolated data points (i.e., records in which at least one of the color markers had interpolated rather than directly captured $x, y$ coordinates, $n=168$ ). This analysis was run for the variables contact, angle, and type of interaction. The resulting weighted kappa coefficients are presented in Figure 4.

The interpolated data achieved as good agreement with the human observer's data as the noninterpolated data in assessing

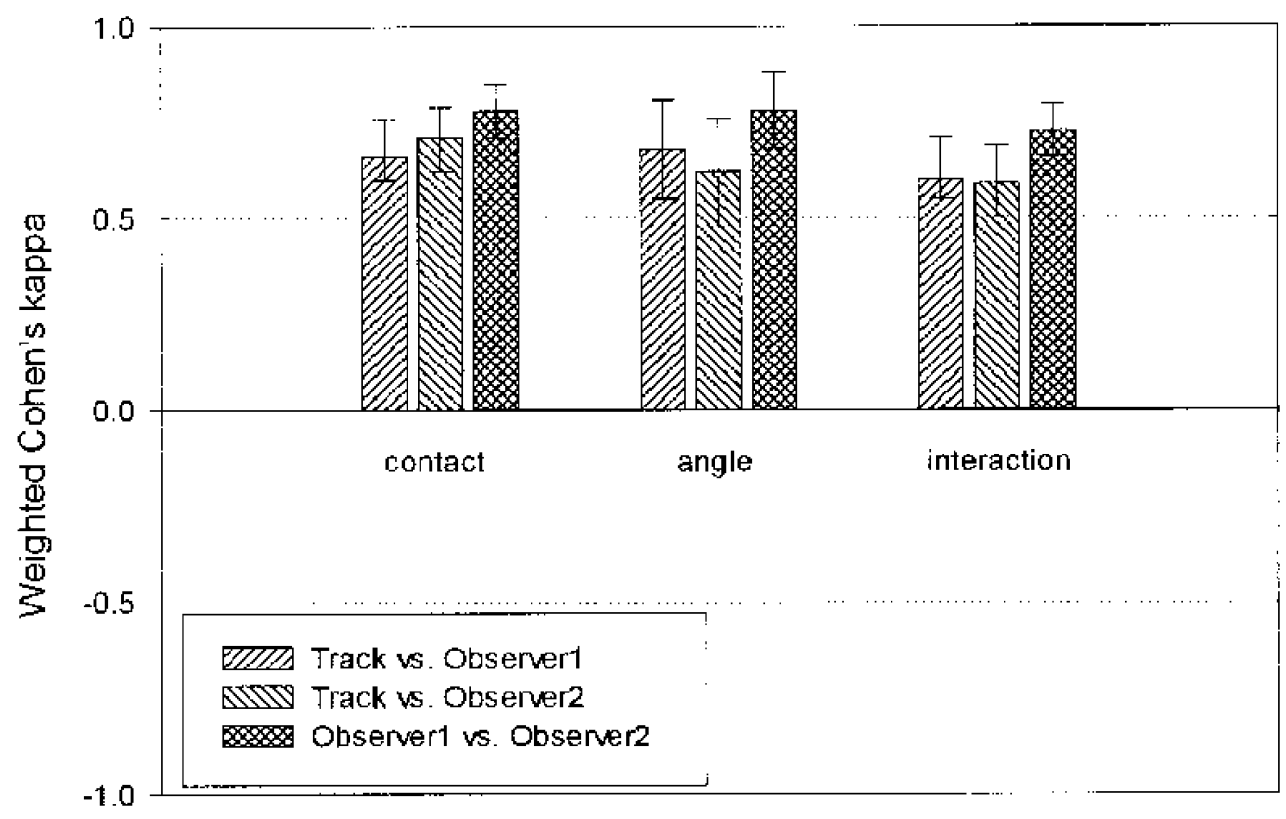

Figure 3. Agreement between automatically tracked data and data obtained manually by 2 independent observers. Weighted Cohen's kappa coefficients $\pm 95 \%$ confidence intervals are displayed. $N=300$ for the variables contact and type of interaction, $N=95$ for the variable angle. 


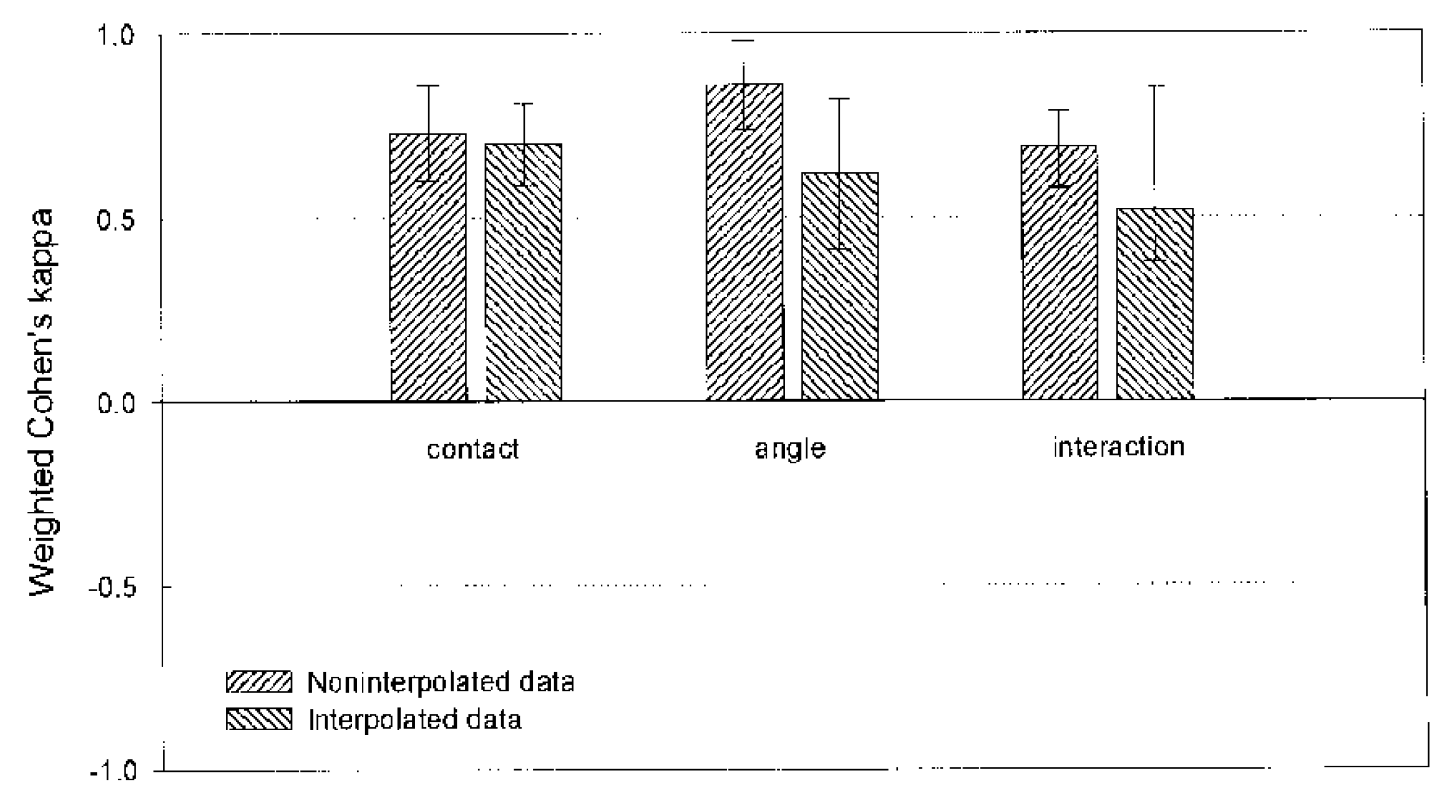

Figure 4. Agreem ent between interpolated and noninterpolated data, obtained automatically by program. Weighted Cohen's kappa coefficients $\pm 95 \%$ confidence intervals are displayed. $N=132$ for noninterpolated data in the variables contact and type of interaction, $N=168$ for interpolated data in the variables contact and type of interaction, $N=61$ for noninterpolated data in the variable angle, $N=47$ for interpolated data in the variable angle.

whether or not there was contact between the 2 pigs. The agreement between the interpolated data and the observer's data was lower than that between the noninterpolated data and the observer's data for the angle and type of interaction variables.

\section{DISCUSSION}

Our automated system is able to perform its two main tasks in a reasonably reliable way. First, it can identify records in which the EthoVision system did not locate the colored marks correctly and replace these with interpolated values. The user of the system can decide whether to use the interpolated records or discard them. This decision involves a tradeoff between the amount of lost data and the reduction of accuracy. In our data set, the interpolation was very successful in assessing whether the pigs were in contact, but was less precise in classifying the mutual positions of the pigs into the correct types of interaction. According to manufacturer, Version 2.0 of EthoVision for Windows should make substantially fewer errors in capturing the positions of colored marks (Spink, Buma, \& Tegelenbosch, 2000). Consequently, the problem of interpolation may become less significant. However, for some detailed sequential analyses of mutual positions, having a gap-free sequence of records may still be important. For this, some interpolation will be necessary even if the errors are less frequent.

Second, our automated system is able, with an accuracy reasonably close to the level of agreement between 2 human observers, to capture the contacts and mutual angles of 2 interacting pigs, thus describing their mutual configurations. Consequently, the system can distin- guish which type of interaction is represented in each scan: an unstable head-to-head position, Pig A or Pig B attacking, resting in the clinch position, or not in contact. Admittedly, the absolute kappa level of about .6 for the classification into interaction types indicates substantial random error. Therefore, it is questionable whether the data acquired with the system can be used for detailed sequential analysis, because if pairs of positions instead of just single instantaneous positions are used, the agreement will be even lower. However, the automatically collected data can certainly be used for assessing treatment or factor effects (e.g., rearing experience, body weight, sex of animal) on the general character of pig interactions. Large data sets can be easily collected, which should allow even subtle effects to be detected despite random tracking and interpreting error.

We shall use our automated system to evaluate whether pigs with different preweaning social experience behave differently in social interactions after weaning. We shall be able to determine whether the frequency of positions such as head-to-head and attack differ when pigs interact with littermates, pigs from other litters that they have met previously, and complete strangers. If we find that the head-to-head position is more frequent in playful interactions than in serious fights, then the ratio of attack versus head-to-head positions could provide a useful measure of the "seriousness" of an interaction. We are also investigating the speed with which pigs differing in preweaning social experience resolve conflicts with aliens. Variables of interest in this analysis include the time spent in the clinch position (which is typical for ex- 
hausted pigs in the middle of an unresolved conflict), the latency to switch from bilateral to unilateral attacks, and the relative frequency of head-to-head and attack positions.

However, the use of the system is not limited to this specific experiment on pigs. With minor adjustments, the system can be used to analyze body orientations and mutual positions of different species of animals in a wide range of situations. If coupled with the new version of EthoVision, its reliability and usefulness may be further enhanced. We can provide a working version of our software to readers interested in adapting it to their specific projects.

\section{REFERENCES}

Fraser, D., Kramer, D. L., Pajor, E. A., \& Weary, D. M. (1995). Conflict and cooperation: Sociobiological principles and the behaviour of pigs. Applied Animal Behaviour Science, 44, 139-157.

Giersing, M., \& Anderson, A. (1998). How does former acquaintance affect aggressive behaviour in repeatedly mixed male and female pigs? Applied Animal Behaviour Science, 59, 297-306.

GolAnI, I. (1992). A mobility gradient in the organization of vertebrate movement: The perception of movement through symbolic language. Behavioral \& Brain Sciences, 15, 249-308.

Golani, I., KafKafi, N., \& Drai, D. (1999). Phenotyping stereotypic behaviour: Collective variables, range of variation and predictability. Applied Animal Behaviour Science, 65, 191-220.
Microsoft Corp. (1998). Visual FoxPro (Version 6.0 PE) [Computer program].

Moran, G., Fentress, J. C., \& Golani. I. (1981). A description of relational patterns of movement during "ritualized fighting" in wolves. Animal Behaviour, 29, 1146-1165.

Newberry, R. C., Śpinka, M., \& Cloutier, S. (2000). Early social experience of piglets affects rate of adaptation to strangers after weaning. In A. Ramos, L. C. Pinheiro Machado Filho, \& M. J. Hötzel (Eds.), Proceedings of the thirty-fourth International Congress of the ISAE, October, 17-20, 2000, Florianópolis, Brazil. Federal University of Santa Catarina, Florianópolis, Brazil.

Noldus Information Technology (1997). EthoVision (Version 1.90) [Reference manual].

Rushen, J., \& PAJOR, E. (1987). Offence and defence in fights between young pigs (Sus scrofa). Aggressive Behaviour, 13, 329-346.

SAS InSTITUTE (2000). The SAS System (Version 2000) [Computer program].

Spink, A. J., Buma, M. O. S., \& Tegelenbosch, R. A. J. (2000). EthoVision color identification: A new method for color tracking using both color and saturation. In Measuring Behavior 2000, Third International Conference on Methods and Techniques in Behavioral Research (pp. 295-297). Nijmegen, The Netherlands: Noldus.

Spink, A. J., Tegelenbosch, R. A. J., Buma, M. O. S., \& Noldus, L. P. J. J. (2001). The EthoVision video tracking system: A tool for behavioral phenotyping of transgenic mice. Physiology \& Behavior, 73, 719-730.

(Manuscript received April 2, 2001; accepted for publication May 22, 2001.) 